\title{
Experimental Study on the Impact of Vehicular Obstructions in VANETs
}

\author{
Rui Meireles ${ }^{1,3}$, Mate Boban ${ }^{2,3}$, Peter Steenkiste ${ }^{1}$, Ozan Tonguz $^{2}$ and João Barros ${ }^{3}$ \\ \{rui@cmu.edu, mboban@cmu.edu, prs@cs.cmu.edu, tonguz@ece.cmu.edu, jbarrosefe.up.pt\} \\ ${ }^{1}$ Department of Computer Science, Carnegie Mellon University, USA \\ ${ }^{2}$ Department of Electrical and Computer Engineering, Carnegie Mellon University, USA \\ ${ }^{3}$ Instituto de Telecomunicações, FEUP DEEC, University of Porto, Portugal
}

\begin{abstract}
Channel models for vehicular networks typically disregard the effect of vehicles as physical obstructions for the wireless signal. We aim to clarify the validity of this simplification by quantifying the impact of obstructions through a series of wireless experiments. Using two cars equipped with Dedicated Short Range Communications (DSRC) hardware designed for vehicular use, we perform experimental measurements in order to collect received signal power and packet delivery ratio information in a multitude of relevant scenarios: parking lot, highway, suburban and urban canyon. Upon separating the data into line of sight (LOS) and non-line of sight (NLOS) categories, our results show that obstructing vehicles cause significant impact on the channel quality. A single obstacle can cause a drop of over $20 \mathrm{~dB}$ in received signal strength when two cars communicate at a distance of $10 \mathrm{~m}$. At longer distances, NLOS conditions affect the usable communication range, effectively halving the distance at which communication can be achieved with $90 \%$ chance of success. The presented results motivate the inclusion of vehicles in the radio propagation models used for VANET simulation in order to increase the level of realism.
\end{abstract}

Index Terms-VANET, vehicle-to-vehicle communication, experiment, radio propagation, channel model, simulation

\section{INTRODUCTION}

Based on the parties involved, two main communication paradigms exist in Vehicular Ad Hoc Networks (VANETs): Vehicle-to-Vehicle (V2V) communication, where vehicles on the road communicate amongst themselves; and Vehicle-toInfrastructure (V2I) communication, where vehicles communicate with nearby roadside equipment. The relatively low heights of the antennas on the communicating entities in $\mathrm{V} 2 \mathrm{~V}$ communication imply that the optical line of sight (LOS) can easily be blocked by an obstruction, either static (e.g., buildings, hills, foliage) or mobile (other vehicles on the road).

There exists a wide variety of experimental studies dealing with the propagation aspects of $\mathrm{V} 2 \mathrm{~V}$ communication. Many of these studies deal with static obstacles, often identified as the key factors affecting signal propagation (e.g., [1], [2], [3]). However, it is reasonable to expect that a significant portion

This work was funded in part by the Portuguese Foundation for Science and Technology under the Carnegie Mellon | Portugal program (grants SFRH/BD/33771/2009 and SFRH/BD/37698/2007) and the DRIVE-IN project (CMU-PT/NGN/0052/2008. http://drive-in.cmuportugal.org).

The authors would like to thank Paulo Oliveira, Xiaohui Wang and Shshank Garg for their help performing the experiments. They would also like to acknowledge the authors of the $\mathrm{R}$ environment for statistical computing [15]. of the $\mathrm{V} 2 \mathrm{~V}$ communication will be bound to the road surface, especially in highway environments, thus making the LOS between two communicating nodes susceptible to interruptions by other vehicles. Even in urban areas, it is likely that other vehicles, especially large public transportation and commercial vehicles such as buses and trucks, will often obstruct the LOS.

Despite this, as noted in [4], virtually all of the state of the art VANET simulators neglect the impact of vehicles as obstacles on signal propagation, mainly due to the lack of an appropriate methodology capable of incorporating the effect of vehicles realistically and efficiently. To that end, a model was designed in [5] which showed that other vehicles often obstruct the LOS between the transmitter and the receiver, thus affecting the received signal power and the packet reception rate. This motivated us to perform extensive measurements to precisely determine the impact of vehicles on the signal power and packet reception rate in different real world scenarios.

Based on the recent experimental V2V studies pointing out that the LOS component of the signal carries the larger portion of the power when compared to reflected/diffracted components [6], [7], we focused on measuring the impact of NLOS conditions on received signal strength and packet delivery ratio. Our goal was to isolate the following three variables:

- Environment - We distinguish one parking lot and three on-the-road scenarios: urban, suburban, and highway. The parking lot experiments allowed us to control factors such as the distance between the vehicles and the number and location of vehicles obstructing the LOS. The on-the-road experiments allowed us to analyze the effect of NLOS conditions in the typical real world environments where VANETs will be used.

- Line of sight conditions - To isolate the impact of moving vehicles on the channel quality, we distinguished between the following situations: LOS, NLOS due to vehicular obstacles, and NLOS due to static obstructions.

- Time of day - We introduce this variable to help determine how often the vehicles encounter NLOS conditions at different times of day and how this affects the signal.

Using these variables and following the work reported in [5], we designed a set of experiments using two vehicles equipped 


\begin{tabular}{|l|c|c|}
\hline Parameter & $\mathbf{8 0 2 . 1 1 p}$ & $\mathbf{8 0 2 . 1 1 b} / \mathbf{g}$ \\
\hline Channel & 180 & 1 \\
\hline Center frequency (MHz) & 5900 & 2412 \\
\hline Bandwidth (MHz) & 20 & 20 \\
\hline Data rate (Mbps) & 6 & 1 \\
\hline Tx power (setting, dBm) & 18 & 18 \\
\hline Tx power (measured, dBm) & 10 & 16 \\
\hline Antenna gain (dBi) & 5 & 3 \\
\hline Beacon frequency (Hz) & 10 & 10 \\
\hline Beacon size (Byte) & 36 & 64 \\
\hline
\end{tabular}

TABLE I

HARDWARE CONFIGURATION PARAMETERS

with Dedicated Short Range Communication (DSRC) devices to characterize the impact of vehicles as obstacles on V2V communication at the communication link level. We aimed at quantifying the additional attenuation and packet loss due to vehicular obstructions.

The rest of the paper is organized as follows. The experimental setup is described in Section II. Section III discusses the results and Section IV describes previous work on experimental evaluation and modeling of $\mathrm{V} 2 \mathrm{~V}$ communication. Section $\mathrm{V}$ concludes the paper.

\section{EXPERIMENTAL SETUP}

\section{A. Network Configuration}

The experiments were performed with a simple vehicular ad-hoc network comprised of two vehicles, both sedans of similar and average height: a Toyota Corolla and a Pontiac G6. In order to directly affect the line of sight between these two vehicles, we used a larger, non-networked vehicle as a LOS obstacle: a Ford E-Series van. The relevant dimensions of all three vehicles are depicted in Fig. 11. With $26 \mathrm{~cm}$ antennas centrally mounted on the roof for the best possible reception (as experimentally shown by [9]), the van sits around $37 \mathrm{~cm}$ taller than the tip of the antennas on the sedans, effectively blocking the LOS while positioned between them.

We equipped each car with a NEC LinkBird-MX, a custombuilt development platform for vehicular communications [10]. These DSRC devices operate at the 5.85-5.925 GHz band and implement the IEEE $802.11 \mathrm{p}$ wireless standard, specifically designed for automotive use [11]. Adding a GPS receiver to each Linkbird-MX and taking advantage of the built-in beaconing functionality, we recorded the locations of the vehicles, the packet delivery ratio (PDR) and the received signal strength indicator (RSSI) throughout the experiments.

To get a sense of the difference between the IEEE $802.11 \mathrm{p}$ and the off-the-shelf WiFi (IEEE 802.11b/g) equipment, we also performed experiments with Atheros WiFi cards and GPS receivers. We used the ping application and the Wireshark network protocol analyzer [12] to collect the same location, PDR, and RSSI information as with the Linkbirds.

The hardware configuration parameters used in the experiments are summarized in Table [1 We used the lowest available data rate for each standard to get the largest possible communication range. The actual power at the antenna outputs was measured using a real time spectrum analyzer and no significant power fluctuations were observed. We used $20 \mathrm{MHz}$ channels for both standards to have a closer comparison of the two. Relatively small packet sizes (see Table I) were used in order to reflect the message size for proposed safety applications [13]. Since larger packets would be more susceptible to fading, our results provide a lower bound on the effect of non-line of sight conditions.

\section{B. Scenarios}

A set of parking lot and on-the-road experiments were designed to isolate the effect of vehicles as obstacles from other variables and to provide insights into the effect of vehicles in different environments where VANETs will be used. All of the experiments were performed in, or near, Pittsburgh PA, USA in good weather conditions, with clear skies and no rain.

The parking lot experiments were performed in the Loews Complex parking lot (lat: 40.405139, long: -79.91925), which is open, large (200 m by $200 \mathrm{~m}$ ), mostly flat and during the day, practically empty. We collected signal information for the following scenarios:

- Cars parked 10, 50 and $100 \mathrm{~m}$ apart, with and without the van placed halfway across the gap.

- Cars starting next to each other and slowly moving apart, with and without an obstruction in between them. In this experiment, we replaced the obstructing van with a 4 meter tall semi-trailer truck shown in Fig. 2(c).

For the on-the-road experiments, we identified three typical environments where VANETs will be used:

- Highway - In this environment, the obstructions are caused by the terrain profile, e.g., crests and corners. We performed experiments on a $85 \mathrm{~km}$ stretch of the U.S. Interstate 79 between the Pittsburgh Airport (lat: 40.4516, long: -80.1099) and Grove City, PA (lat: 41.14174, long: 80.15498).

- Suburban - In this environment, wide streets are typically lined with small buildings and trees. There are also occasional crests, dips, and blind corners. We used a residential, 4 lane, $5 \mathrm{~km}$ stretch of Fifth Ave. in Pittsburgh, PA (lat: 40.45008, long: -79.92768) for this scenario.

- Urban canyon - In this environment, streets cut through dense blocks of tall buildings which significantly affect the reception of radio signals. We performed experiments on a two km trapezoidal route around Grant Street (lat: 40.44082, long: -79.99579) in downtown Pittsburgh (Fig. 2(b)).

For each environment, we performed the experiments by driving the cars for approximately one hour, all the time collecting GPS and received signal information. Throughout the experiment, we videotaped the view from the car following in the back for later analysis of the LOS/NLOS conditions.

We performed two one-hour experiment runs for each onthe-road scenario: one at a rush hour period with frequent 


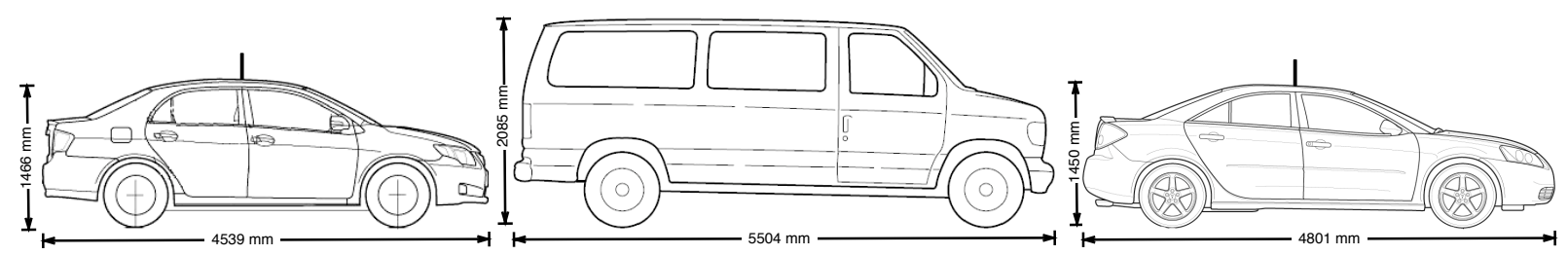

Fig. 1. Scaled drawing of the vehicles used in the experiments. Left to right we have a 2009 Toyota Corolla, a 2010 Ford E-Series, and a 2009 Pontiac G6. Blueprints courtesy of carblueprints.info [8].

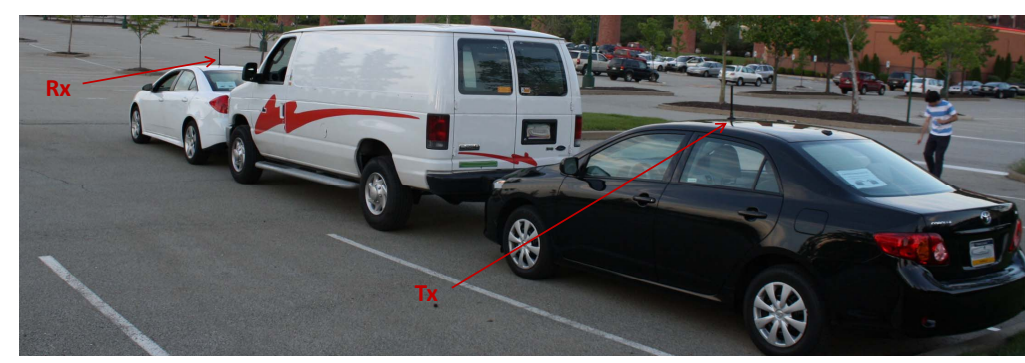

(a) Parking lot environment: experiment with the obstructing van

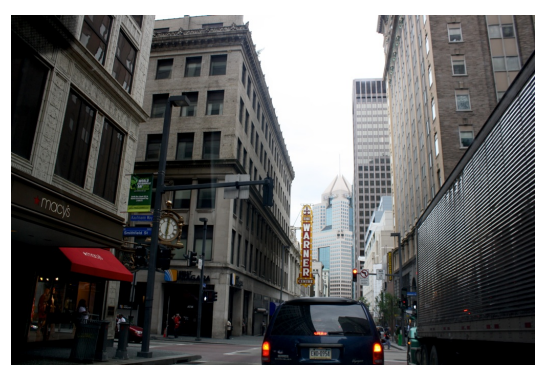

(b) Urban canyon in Downtown Pittsburgh

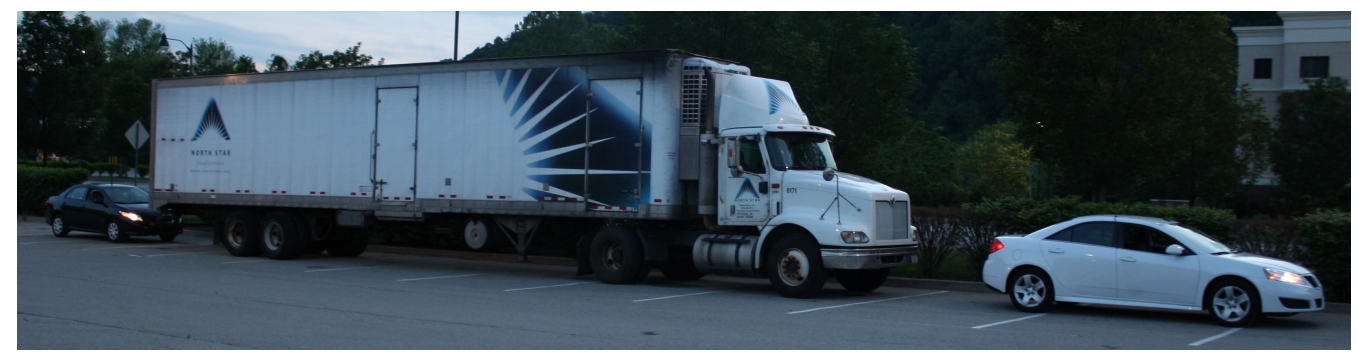

(c) Parking lot environment: experiment with the obstructing truck

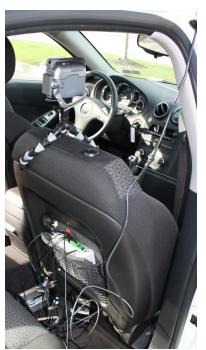

(d) Hardware

Fig. 2. Experimental setup.

NLOS conditions, and the other late at night, when the number of vehicles on the road (and consequently, the frequency of vehicle-induced NLOS conditions) is substantially lower. This, by itself, worked as a heuristic for the LOS conditions. Furthermore, to more accurately distinguish between LOS and NLOS conditions, we used the recorded videos to separate the LOS and NLOS data.

To help analyze the experiments in detail, we wrote a web-based visualization suite (Fig. 3) that can be used to replay the experiments and observe: i) the movement of the communicating vehicles on the road overlaid on a map; ii) the video recorded from the trailing car and, iii) RSSI, PDR and distance information. The visualization tool as well as all the collected data are freely available on our website [14].

\section{RESULTS}

\section{A. Parking lot experiments}

All of the parking lot experiments were performed at relatively short distances, meaning the packet delivery ratio was almost always $100 \%$. We therefore focus on RSSI to analyze the effect of LOS conditions on channel quality. For ease of presentation, we report the RSSI values in $\mathrm{dB}$ as provided by

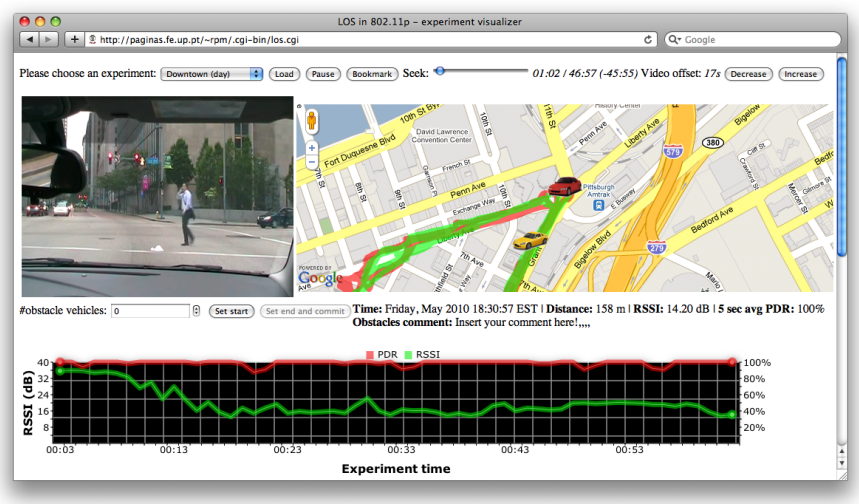

Fig. 3. Experiment visualization software.

the Atheros cards. The RSSI values can be converted to $\mathrm{dBm}$ by subtracting 95 from the presented values.

First, we consider the experiments where the cars were placed at a fixed distance from each other. Figure 4 shows the 


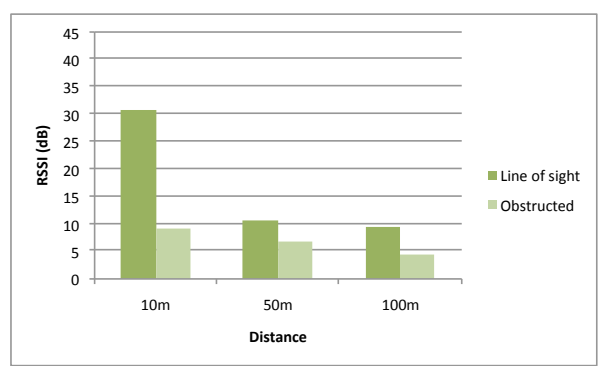

(a) $802.11 \mathrm{~g}$

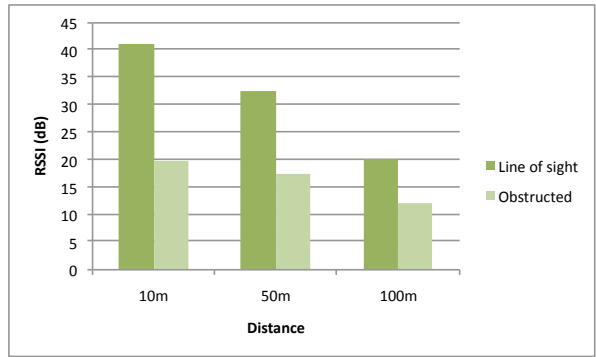

(b) $802.11 \mathrm{p}$

Fig. 4. Parking lot experiment: average received signal strength measured at fixed distances with and without the obstructing van for both $802.11 \mathrm{~g}$ and $802.11 \mathrm{p}$ standards.

RSSI results. The standard deviation was under $1 \mathrm{~dB}$ and the 95\% confidence intervals were too small to represent; we thus focus on the average values. The difference in absolute RSSI values between the $802.11 \mathrm{~b} / \mathrm{g}$ and $802.11 \mathrm{p}$ standards is mainly due to the difference in antenna gains, hardware calibrations, and the quality of the radios.

Blocking the LOS has clear negative effects on the RSSI. Even though the absolute values differ between the standards, the overall impact of NLOS conditions is quite similar. At $10 \mathrm{~m}$, the van reduced the RSSI by approximately $20 \mathrm{~dB}$ in both cases. As the distance between communicating nodes increased, the effect of the van was gradually reduced. At $100 \mathrm{~m}$, the RSSI in the NLOS case was approximately 5 and $7 \mathrm{~dB}$ below the LOS case for $802.11 \mathrm{~b} / \mathrm{g}$ and $802.11 \mathrm{p}$, respectively.

Furthermore, we performed an experiment where, starting with the cars next to each other, we slowly moved them apart. We did this experiment without any LOS obstruction and with a $4 \mathrm{~m}$ tall semi-trailer truck parked halfway between the vehicles (Fig. 2(c)). Figure 5 shows the RSSI as a function of distance. The dots represent individual samples, while the curves show the result of applying locally weighted scatter plot smoothing (LOWESS) to the individual points. The truck had a large impact on RSSI, with a loss of approximately $27 \mathrm{~dB}$ at the smallest recorded distance of $26 \mathrm{~m}$ (the length of the truck) when compared with the LOS case. For comparison, the van attenuated the signal by $12 \mathrm{~dB}$ at $20 \mathrm{~m}$. The RSSI drop caused by the truck decreased as the cars move further away from it, an indication that the angle of the antennas' field of view that gets blocked makes a difference.

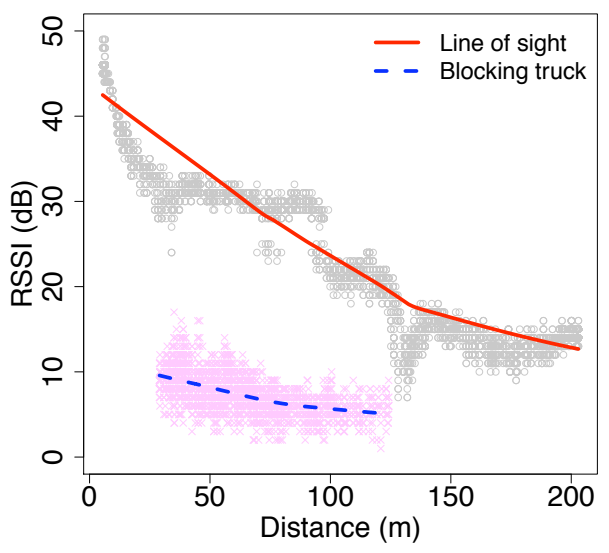

Fig. 5. RSSI as a function of distance in 802.11p for LOS and NLOS conditions due to the obstructing truck shown in Fig. 2(c)

\section{B. On-the-road experiments}

For the on-the-road experiments, we drove the test vehicles in the three scenarios identified in Section $\Pi-\mathrm{B}$ and collected RSSI and PDR information to use as indicators of channel quality. To accurately analyze the LOS and NLOS conditions, we placed each data point in one of the following line of sight categories, according to the information we obtained by reviewing the experiment videos:

- Line of sight (LOS) - no obstacles between the sender and receiver vehicles.

- Vehicular obstructions (NLOS-VO) — LOS blocked by other vehicles on the road.

- Static obstructions (NLOS-SO) - LOS blocked by immovable objects, such as buildings or terrain features, like crests and hills.

To compute the PDR, we counted the number of beacons sent by the sender and the number of beacons received at the receiver in a given time interval. We used a granularity of 5 seconds (50 beacons) for the calculations. We use $10 \mathrm{~m}$ bins for the distance and show: the mean, its associated $95 \%$ confidence intervals and the 20 and $80 \%$ quantiles (dashed lines). To make the data easier to read, we use LOWESS to smooth the curves.

Figure 6 shows the PDR as a function of distance separately for each on-the-road scenario, as well as aggregated over all three. For all scenarios, the PDR for the LOS case is above $80 \%$ even at long distances, only dropping below that threshold in the suburban scenario and only after $400 \mathrm{~m}$. At short distances, the difference between the PDR for LOS and NLOS-VO is almost non-existent. However, above $100 \mathrm{~m}$ there is a significant increase in the number of dropped packets in the NLOS-VO case. In the suburban scenario, the NLOS-VO PDR drops to zero at $500 \mathrm{~m}$. In the urban canyon case, it drops to $30 \%$ at roughly the same distance. Interestingly, in the highway scenario the NLOS-VO PDR stays high at long distances. One possible explanation could be that in the long sweeping highway curves the angle of the antennas' field of view blocked by vehicular obstructions is smaller than in other 


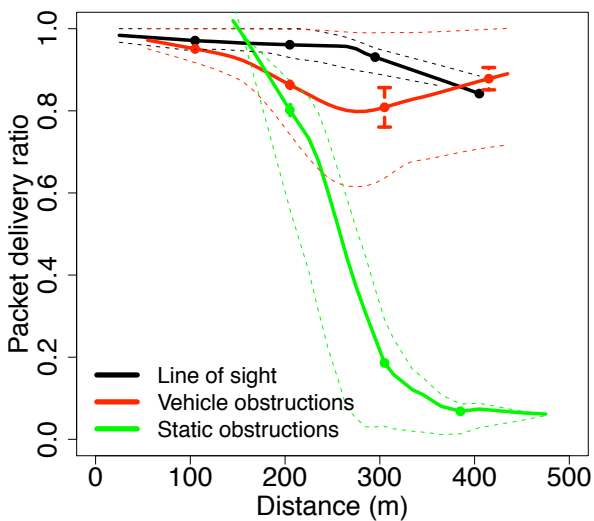

(a) Highway

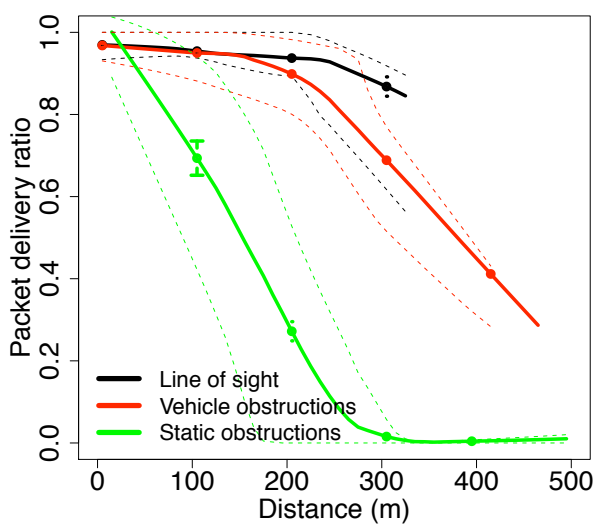

(c) Urban canyon

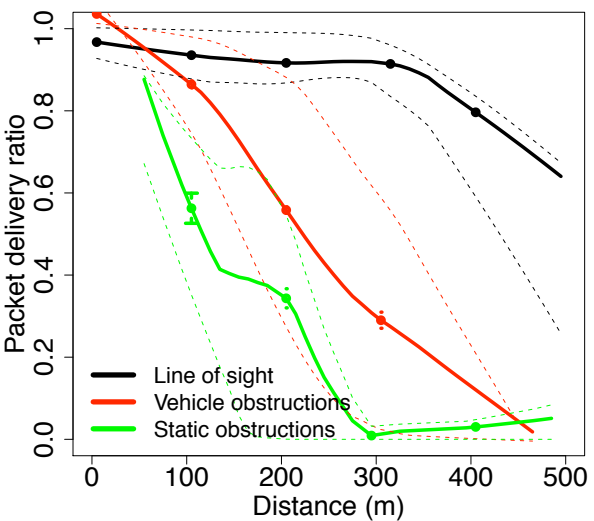

(b) Suburban

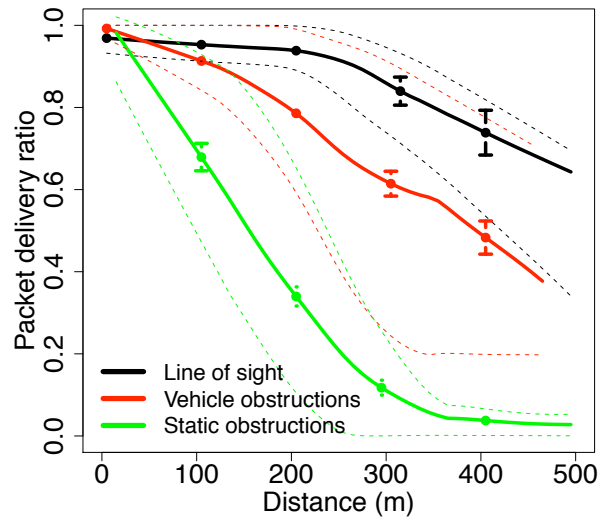

(d) Overall

Fig. 6. Packet delivery ratio as a function of distance for the on-the-road experiments. The dashed lines represent the $20 \%$ and $80 \%$ quantiles.

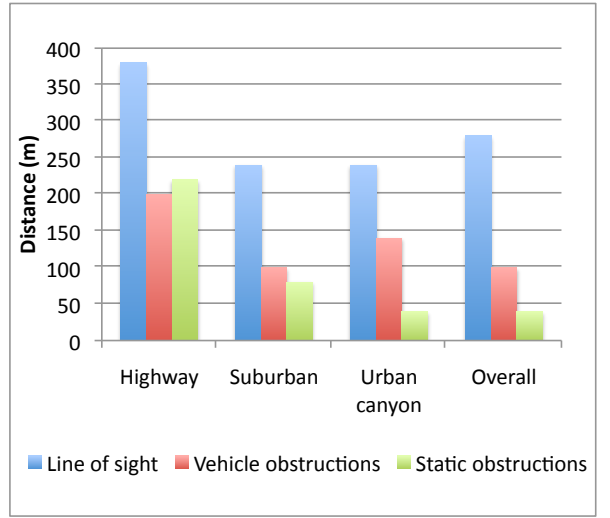

Fig. 7. The reliable communication range calculated as the maximum distance at which the PDR was above $90 \%$.

environments. Looking at the data for the static obstructions, we see marked differences in PDR, even when compared to the NLOS-VO case. In all environments, the PDR drops to $20 \%$ or less at approximately $300 \mathrm{~m}$, including the highway environment.

To shed some light on the practical implications of these results, Fig. 7 shows the reliable communication range under different LOS conditions. This range was calculated as the

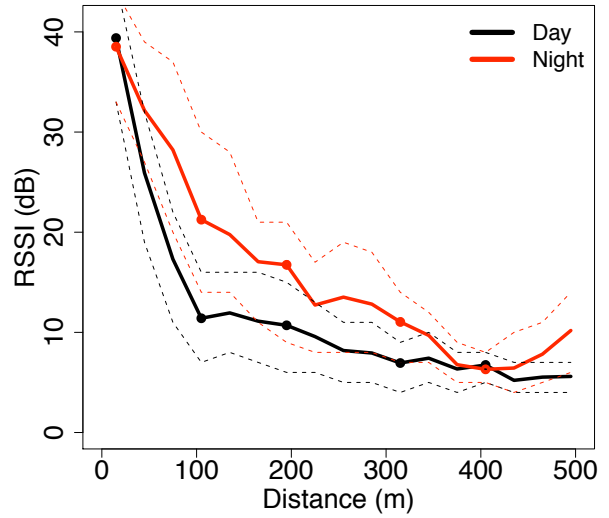

Fig. 8. The overall difference between the daytime experiments (frequent NLOS conditions) and nighttime experiments (predominantly LOS).

maximum distance at which the mean PDR was above or equal to $90 \%$. In all of the environments, the obstructing vehicles significantly decreased the effective communication range. The largest relative difference was observed in the suburban environment, with a $60 \%$ reduction in range, and the smallest in the urban environment, with a $40 \%$ reduction. The static obstructions have an even more negative impact, decreasing the overall communication range by $85 \%$ on average. Using 

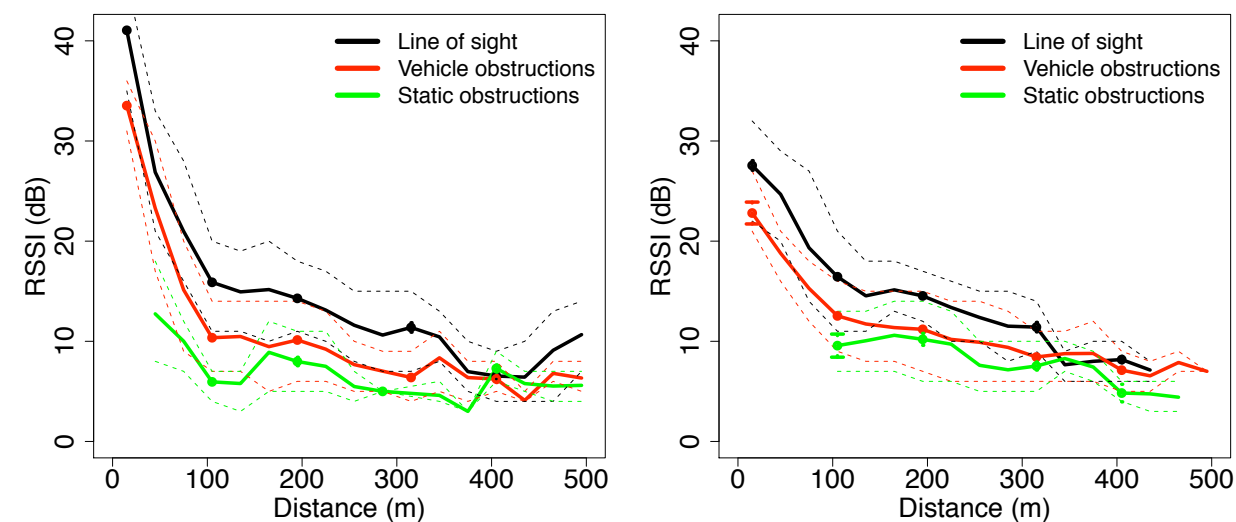

(a) Suburban (35.000 LOS, 20.000 NLOS-VO and (b) Highway (14.000 LOS, 17.000 NLOS-VO and 7.000 NLOS-SO data points) 1.000 NLOS-SO data points)
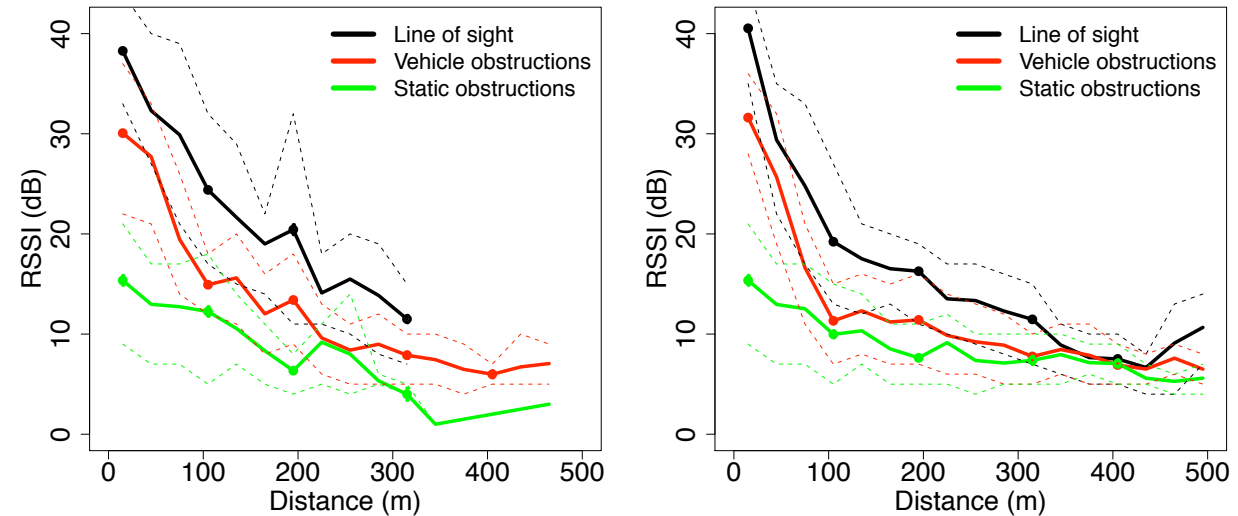

(c) Urban canyon (62.000 LOS, 25.000 NLOS-VO and (d) Overall (35.000 LOS, 62.000 NLOS-VO and 9.000 1.000 NLOS-SO data points)

NLOS-SO data points)

Fig. 9. Received signal strength as a function of distance for the on-the-road experiments. The dashed lines represent the $20 \%$ and $80 \%$ quantiles.

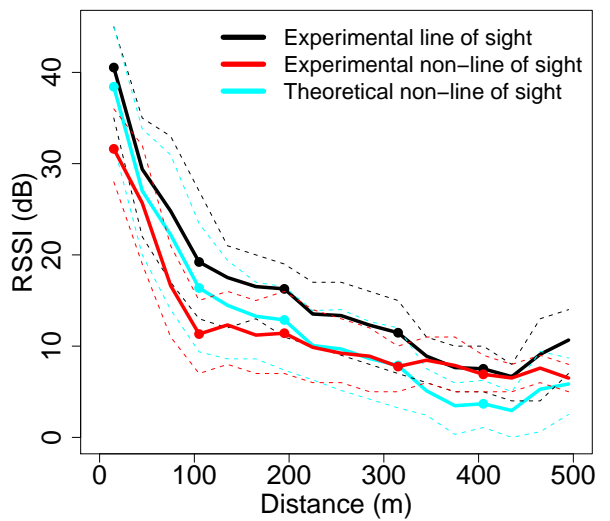

Fig. 10. Attenuation as computed by the theoretical knife-edge model compared against the experimentally obtained data (188.000 LOS and 62.000 NLOS-VO data points).

other target success probabilities (from 95\% to 50\%), we observed the following trends:

- For targets above $90 \%$, the importance of the LOS conditions is reduced. For a target PDR of $95 \%$, NLOS conditions cause a $25 \%$ decrease of the usable range.

- Gradually decreasing the target PDR from $90 \%$ to $50 \%$ we observed a trend where the effective range in NLOSVO conditions converges to around $50 \%$ of what is achievable in the LOS case.

Regarding RSSI, we analyze each successfully received packet and plot the mean RSSI as a function of distance using 30 meter bins. We also plot $20 \%$ and $80 \%$ quantiles and $95 \%$ confidence intervals at selected points.

Figure 8 shows the overall RSSI as a function of distance for daytime (frequent NLOS) and nighttime (infrequent NLOS) experiments. Since the same routes were used in both experiments, the obstructing vehicles were the only variable changing between day and night. The difference between the plots shows the significant impact of the obstructing vehicles on the received signal power.

Figure 9 shows the resulting RSSI plots for each of the individual on-the-road experiment scenarios (Figs. 9(a)-(c)) and for the general case where we aggregate all data in each LOS category (Fig. 9(d)). The difference between LOS and NLOS-VO conditions varies in magnitude across scenarios but the overall trends are roughly similar and indicative of 
the significant impact that both vehicles and static obstacles had. Generally, we can observe the following trends in the difference between LOS and NLOS-VO conditions as we move from short to longer distances:

1) There is a large average difference of up to $10 \mathrm{~dB}$ between LOS and NLOS-VO conditions at short distances. This is most likely due to the vehicles blocking a large angle of the antennas' field of view. In the parking lot experiments the difference was up to $20 \mathrm{~dB}$ at these distances (see Fig. 4). The smaller difference in the road experiments is due to the fact that we are averaging out over all vehicular obstructions, regardless of their height or angle relative to the antennas. Interestingly, the absolute RSSI values at short distances in the highway scenario were significantly lower than in the other scenarios.

2) As the distance increases, the difference between LOS an NLOS-VO conditions decreases slightly and then roughly stabilizes.

3) At longer distances (above approximately $400 \mathrm{~m}$ ), the difference gradually decreased to the point of being nonexistent. This can be explained by two factors. First, the successful packet reception requires a minimum SINR. If the attenuation is strong enough that this threshold is crossed, the packet is dropped. At long distances, the successfully received packets are close to this minimum SINR threshold, so the difference between LOS conditions can only be observed in terms of PDR. Also, for $5.9 \mathrm{GHz}$ frequency and the heights of the antennas, the first Fresnel ellipsoid becomes significantly obstructed by the ground level at $400 \mathrm{~m}$ [16, Chap. 3]. Therefore, the road itself starts effectively blocking the LOS between the communicating vehicles. This finding is in line with the results reported in [5].

It is interesting to observe the large difference in RSSI observed in the urban canyon scenario. This difference is perhaps best explained by the multipath effects caused by the buildings. The tunneling effect created reflected rays with relatively low phase difference to the LOS ray, which in turn acted constructively on the received power.

Figure 10 compares the obtained experimental results against the NLOS-induced attenuation predicted by the theoretical knife-edge model. For each experimental data point collected in the LOS category, we placed a vehicle obstacle uniformly at random between the sender and receiver and computed the resulting RSSI according to the knifeedge model [17]. The obstacles' dimensions were taken from the best fit distributions reported in [5]. Figure [10 shows that the knife-edge model underestimated the attenuation at shorter distances and overestimated it at distances closer to the maximum communication range. This can be explained by the assumption of the knife edge model that the only factor affecting the signal is the obstacle in consideration. While this would be the case for free space, in the real world environments the surrounding terrain and constructions also have a role to play.

We also captured data pertaining to the effect of static obstructions on the channel quality. In the urban canyon the obstructions were mainly buildings, which had a profound impact on RSSI. A loss of around $15 \mathrm{~dB}$ compared with the NLOS-VO case at shorter distances and around $4 \mathrm{~dB}$ at larger distances was observed. In the suburban and highway scenarios, obstructions were mostly created by crests on the road. The results indicate that they can make a difference of up to $3 \mathrm{~dB}$ of additional attenuation atop the NLOS-VO attenuation.

The results presented in this section inevitably point to the fact that obstructing vehicles have to be accounted for in channel modeling. Not modeling the vehicles results in overly optimistic received signal power, PDR and communication range.

\section{RELATED WORK}

Regarding V2V communication, Otto et al. in [1] performed $\mathrm{V} 2 \mathrm{~V}$ experiments in the $2.4 \mathrm{GHz}$ frequency band in an open road environment and reported a significantly worse signal reception during a traffic heavy, rush hour period in comparison to a no traffic, late night period. A similar study presented in [18] analyzed the signal propagation in "crowded" and "uncrowded" highway scenarios (based on the number of vehicles on the road) for the $60 \mathrm{GHz}$ frequency band, and reported significantly higher path loss for the crowded scenarios.

With regards to experimental evaluation of the impact of vehicles and their incorporation in channel models, a lightweight model based on Markov chains was proposed in [19]. Based on experimental measurements, the model extends the stochastic shadowing model and aims at capturing the time-varying nature of the $\mathrm{V} 2 \mathrm{~V}$ channel based on a set of predetermined parameters describing the environment. Tan et al. [20] performed experimental measurements in various environments (urban, rural, highway) at $5.9 \mathrm{GHz}$ to determine the suitability of DSRC for vehicular environments with respect to delay spread and Doppler shift. The paper distinguishes LOS and NLOS communication scenarios by coarsely dividing the overall obstruction levels. The results showed that DSRC provides satisfactory performance of the delay spread and Doppler shift, provided that the message is below a certain size. A similar study was reported in [7], where experiments were performed at 5.2 GHz. Path loss, power delay profile, and Doppler shift were analyzed and statistical parameters, such as path loss exponent, were deduced for given environments. Based on measurements, a realistic model based on optical ray tracing was presented in [21]. The model encompassed all of the obstructions in a given area, including the vehicles, and yielded results comparable with the real world measurements. However, the high realism that the model exhibits is achieved at the expense of high computational complexity.

Experiments in urban, suburban, and highway environments with two levels of traffic density (high and low) were reported in [22]. The results showed significantly differing channel 
properties in low and high traffic scenarios. Based on the measurements, several $\mathrm{V} 2 \mathrm{~V}$ channel models were proposed. The presented models are specific for a given environment and vehicle traffic density. Several other studies [23], [24], [25], [26] point out that other vehicles apart from the transmitter and receiver could be an important factor in modeling the signal propagation by obstructing the LOS between the communicating vehicles.

Virtually all of the studies mentioned above emphasize that LOS and NLOS for V2V communication have to be modeled differently, and that vehicles act as obstacles and affect signal propagation to some extent. However, these studies at most quantify the macroscopic impact of the vehicles by defining $\mathrm{V} 2 \mathrm{~V}$ communication environments as uncrowded (LOS) or crowded (NLOS), depending on the relative vehicle density, without analyzing the impact that obstructing vehicles have on a single communication link.

\section{CONCLUSiOnS}

In this work we set out to experimentally evaluate the impact of obstructing vehicles on V2V communication. For this purpose, we ran a set of experiments with near-production $802.11 \mathrm{p}$ hardware in a multitude of relevant scenarios: parking lot, highway, suburban and urban canyon.

Our results indicate that vehicles blocking the line of sight significantly attenuate the signal when compared to line of sight conditions across all scenarios. Also, the effect appears to be more pronounced the closer the obstruction is to the sender, with over $20 \mathrm{~dB}$ attenuation at bumper-to-bumper distances. The additional attenuation decreased the packet delivery ratio at longer distances, halving the effective communication range for target average packet delivery ratios between $90 \%$ and $50 \%$. The effect of static obstacles such as buildings and hills was also analyzed and shown to be even more pronounced than that of vehicular obstructions.

With respect to channel modeling, even the experimental measurements proposed for certification testing of DSRC equipment [27] do not directly address the effect of vehicles in the $\mathrm{V} 2 \mathrm{~V}$ environment, thus potentially underestimating the attenuation and packet loss. Our work shows that not modeling the vehicles as physical obstructions takes away from the realism of the channel models, thus affecting the simulation of both the physical layer and the upper layer protocols.

\section{REFERENCES}

[1] J. S. Otto, F. E. Bustamante, and R. A. Berry, "Down the block and around the corner - the impact of radio propagation on inter-vehicle wireless communication," in Proc. of IEEE International Conference on Distributed Computing Systems (ICDCS), 2009.

[2] L. Cheng, B. Henty, D. Stancil, F. Bai, and P. Mudalige, "Mobile vehicleto-vehicle narrow-band channel measurement and characterization of the $5.9 \mathrm{GHz}$ dedicated short range communication (DSRC) frequency band," IEEE JSAC, vol. 25, no. 8, pp. 1501-1516, Oct. 2007.

[3] J. Andersen, T. Rappaport, and S. Yoshida, "Propagation measurements and models for wireless communications channels," IEEE Communications Magazine, vol. 33, no. 1, pp. 42-49, Jan 1995.

[4] F. J. Martinez, C. K. Toh, J.-C. Cano, C. T. Calafate, and P. Manzoni, "A survey and comparative study of simulators for vehicular ad hoc networks (VANETs)," Wireless Communications and Mobile Computing, 2009.
[5] M. Boban, T. T. V. Vinhoza, J. Barros, M. Ferreira, and O. K. Tonguz, "Impact of vehicles as obstacles in vehicular ad hoc networks," IEEE Journal on Selected Areas in Communications, vol. 29, no. 1, January 2011.

[6] G. Acosta and M. Ingram, "Model development for the wideband expressway vehicle-to-vehicle $2.4 \mathrm{GHz}$ channel," in IEEE Wireless Communications and Networking Conference, 2006. WCNC 2006., vol. 3 , April 2006, pp. 1283-1288.

[7] A. Paier, J. Karedal, N. Czink, H. Hofstetter, C. Dumard, T. Zemen, F. Tufvesson, A. Molisch, and C. Mecklenbrauker, "Car-to-car radio channel measurements at $5 \mathrm{GHz}$ : Pathloss, power-delay profile, and delay-Doppler spectrum," in 4th International Symposium on Wireless Communication Systems, 2007. ISWCS 2007., Oct. 2007, pp. 224-228.

[8] "Car blueprints database." [Online]. Available: http://carblueprints.info

[9] S. Kaul, K. Ramachandran, P. Shankar, S. Oh, M. Gruteser, I. Seskar, and T. Nadeem, "Effect of antenna placement and diversity on vehicular network communications," in Proc. IEEE SECON., June 2007, pp. 112 121.

[10] A. Festag, R. Baldessari, W. Zhang, L. Le, A. Sarma, and M. Fukukawa, "Car-2-x communication for safety and infotainment in europe," NEC Technical Journal, vol. 3, no. 1, 2008.

[11] “IEEE Draft Standard IEEE P802.11p,” Tech. Rep., June 2010.

[12] G. Combs et al., "Wireshark-network protocol analyzer." [Online]. Available: http://www.wireshark.org

[13] F. Bai, T. Elbatt, G. Hollan, H. Krishnan, and V. Sadekar, "Towards characterizing and classifying communication-based automotive applications from a wireless networking perspective," 1st IEEE Workshop on Automotive Networking and Applications (AutoNet), 2006.

[14] "802.11p Line of Sight Experiment website." [Online]. Available: http://drive-in.cmuportugal.org/los

[15] $\mathrm{R}$ Development Core Team, R: A Language and Environment for Statistical Computing, R Foundation for Statistical Computing, Vienna, Austria, 2010, ISBN 3-900051-07-0. [Online]. Available: http://www.R-project.org

[16] T. S. Rappaport, Wireless Communications: Principles and Practice. Prentice Hall, 1996.

[17] ITU-R, "Propagation by diffraction," International Telecommunication Union Radiocommunication Sector, Geneva, Recommendation P.526, Feb. 2007.

[18] S. Takahashi, A. Kato, K. Sato, and M. Fujise, "Distance dependence of path loss for millimeter wave inter-vehicle communications," in Proc. IEEE 58th Vehicular Technology Conference (VTC 2003-Fall), vol. 1, Oct. 2003, pp. 26-30.

[19] D. Dhoutaut, A. Regis, and F. Spies, "Impact of radio propagation models in vehicular ad hoc networks simulations," VANET 06: Proceedings of the 3rd international workshop on Vehicular ad hoc networks, pp. 69-78, 2006.

[20] I. L. Tan, W. Tang, K. P. Laberteaux, and A. Bahai, "Measurement and analysis of wireless channel impairments in DSRC vehicular communications," in ICC. IEEE, 2008, pp. 4882-4888.

[21] J. Maurer, T. Fugen, T. Schafer, and W. Wiesbeck, "A new intervehicle communications (ivc) channel model," in Vehicular Technology Conference, 2004. VTC2004-Fall. 2004 IEEE 60th, vol. 1, Sept. 2004, pp. $9-13$ Vol. 1.

[22] I. Sen and D. Matolak, "Vehicle-Vehicle Channel Models for the 5-GHz Band," IEEE Transactions on Intelligent Transportation Systems, vol. 9, no. 2, pp. 235-245, June 2008.

[23] M. Jerbi, P. Marlier, and S. M. Senouci, "Experimental assessment of V2V and I2V communications," in Proc. IEEE Internatonal Conference on Mobile Adhoc and Sensor Systems (MASS 2007), Oct. 2007, pp. 1-6.

[24] H. Wu, M. Palekar, R. Fujimoto, R. Guensler, M. Hunter, J. Lee, and J. Ko, "An empirical study of short range communications for vehicles," in Proc. of the 2nd ACM International workshop on Vehicular ad hoc networks, 2005, pp. 83-84.

[25] D. Matolak, I. Sen, W. Xiong, and N. Yaskoff, " $5 \mathrm{GHz}$ wireless channel characterization for vehicle to vehicle communications," in Proc. IEEE Military Communications Conference (MILCOM 2005), vol. 5, Oct. 2005, pp. 3016-3022.

[26] "Vehicle Safety Communications Project, Final Report," U.S. Department of Transportation, NHTSA, Crash Avoidance Metrics Partnership, Tech. Rep. DOT HS 810 591, 2006.

[27] G. Acosta-Marum and M. Ingram, "Six time- and frequency- selective empirical channel models for vehicular wireless lans," IEEE Vehicular Technology Magazine, vol. 2, no. 4, pp. 4-11, dec. 2007. 